\title{
Towards Another Scientific Revolution
}

\author{
Sönke Bartling and Sascha Friesike
}

But even within those limits, the openness I am advocating would be a giant cultural shift in how science is done, a second Open Science revolution extending and completing the first Open Science revolution, of the 17th and 18th centuries.

-Michael Nielsen

\begin{abstract}
In this introductory chapter we establish a common understanding of what are and what drives current changes in research and science. The concepts of Science 2.0 and Open Science will be introduced. As such we provide a short introduction to the history of science and knowledge dissemination. We explain the origins of our scientific culture which evolved around publication methods. Interdependencies of current concepts will be elucidated and it will be stated that the transition towards Open Science is a complex cultural change. Reasons as to why the change is slow are discussed and the main obstacles are identified. Next, we explain the recent changes in scientific workflows and how these cause changes in the system as a whole. Furthermore, we provide an overview on the entire book and explain what can be found in each chapter.
\end{abstract}

Nicole Forster's goal as a researcher is to enhance cancer treatment. That is why she and her colleagues in the laboratory of Leif W. Ellisen at Massachusetts General Hospital Cancer Center in Boston, Massachusetts, study tumors on individual levels and search for cancer causes. In March 2012 Forster was trying to isolate ribonucleic acid (RNA) - the genetic blueprint for proteins within the cell—within mouse cells. To prepare the cells for her experiment she mixed them with a special gel that provided them with all the nutrients to grow and proliferate, even outside the body, for a short period of time. Yet in the following step, she had to get rid of the gel to get to the information she needed: the RNA. And therein lay

\author{
S. Bartling $(\bowtie)$ \\ German Cancer Research Center, Heidelberg, Germany \\ e-mail: soenkebartling@gmx.de \\ S. Bartling \\ Institute for Clinical Radiology and Nuclear Medicine, Mannheim University \\ Medical Center, Heidelberg University, Mannheim, Germany \\ S. Friesike \\ Alexander von Humboldt Institute for Internet and Society, Berlin, Germany \\ e-mail: friesike@hiig.de
}


her problem. She had never done that specific isolation before and hence did not know how to do it. Her colleagues did not know, either. No one in my lab or even on my floor of the Cancer Center was doing such experiments, said Forster. She was stuck. Then Forster thought of turning to the community of ResearchGate. ResearchGate is a social network (Boyd \& Ellison 2007) for scientists to exchange ideas, publications, and to discuss research. Forster had first signed up to ResearchGate in 2009. She had heard about the network at a conference in Boston and was intrigued: I thought that sharing research experience and discussing topics that you always wanted to discuss with someone would be a great opportunity. I like that it is a professional network where you can help other people and be helped. Since then she had answered multiple questions from fellow ResearchGate members and now it was her turn to ask the community for help. Within $24 \mathrm{~h}$ Forster had a solution. Two researchers replied to her post and suggested different methods. She tried one and it worked. You don't have to search for the best approach via Google or go through all of these publications, Forster says. A social network for scientists helped Forster to solve a problem that she had bugged colleagues about for several weeks within a single day. Forster's case is far from uncommon. Researchers all over the world use modern communication tools such as social networks, blogs, or Wikipedia to enhance their scientific expertise, meet experts, and discuss ideas with people that face similar challenges. They do not abandon classical means of scientific communication such as publications or conferences, but rather they complement them. Today we can see that these novel communication methods are becoming more and more established in the lives of researchers; we argue that they may become a significant part of the future of research. We undertook this book in order to highlight the different developments that are currently arising in the world of knowledge creation. We do not know whether all of these developments will prevail, yet we are certain that institutional knowledge creation will change drastically over the next decade. Naturally, anyone involved in research does well to inform themselves about these developments. There is no perfect way by which research will be carried out in the future. Every researcher has to decide for themselves which technologies and methods they will include in their work. This, however,-as anything in research-starts with informing oneself about what is already out there; it is our goal to provide that information with this book.

\section{Knowledge Creation and Dissemination: A Brief History}

In an early draft-version of this book, the present section was called 'A Brief History of Science'. Yet, we ran into several problems with this heading. Firstly, there is a singularity in the English language that differentiates between knowledge creation that is concerned with the rules of the natural world (science) and knowledge creation that is concerned with the human condition (humanities). Throughout the preparation of this book we constantly ran into this dilemma and 
we would like to take the opportunity to tell you that whenever we talk about science we mean any organized form of knowledge creation (see chapter Open Science and the Three Cultures: Expanding Open Science to all Domains of Knowledge Creation). Secondly, science is often understood as the product created by a scientists. And a scientists is understood as someone with a full-time job at a university or a research institute. Yet, new forms of collaboration reach far beyond our institutional understanding of doing research, which brings us to certain dissent.

As such we labeled the section 'Knowledge Creation and Dissemination'. Knowledge creation and its dissemination are two sides of the same coinknowledge does not impact on society if it is unable to disseminate (Merton 1993). Throughout history we can see that breakthroughs in knowledge creation went hand in hand with breakthroughs in its dissemination. In turn, dissemination is not only bound to technological changes but also societal changes such as freedom of speech or the Renaissance. In large, the present book is a compendium that presents current changes that we see in knowledge creation and dissemination. Actually, many chapters of this book challenge our traditional understanding of how scientific knowledge should be disseminated. Moreover, as of today, researchers' views on how knowledge creation is changing differ drastically in many aspects. And it is likely that our understanding differs from your understanding. As such, all we want to offer in this book is a comprehensive overview on what is changing in the world of knowledge creation, which foundations are being laid today, and what might become essential in the future.

The history of human knowledge is closely linked to the history of civilization-one could even argue that the history of civilization is in large parts based on knowledge creation and its dissemination. In prehistoric times, knowledge was passed from one generation to the next one orally or by showing certain techniques. This mainly applied to basic everyday tasks such as hunting, fire making, manufacturing clothes, or gathering nutritious foods. The creation of this knowledge was not yet structured and it was not recorded, except for occasional drawings like cave paintings. The drastic change in knowledge creation was the invention of a writing system. Roughly at the same time, agriculture came to life. These two inventions combined laid the groundwork for what we today consider civilization. Civilization allowed for the division of labor and hence individuals began to specialize-knowledge creation accelerated. The researcher as a profession concerned with the creation of knowledge made his debut in ancient Greece. Scientists like Plato, Aristotle, Pythagoras, Socrates, or Archimedes wrote their observations down, taught others, and created knowledge that is still relevant roughly 2,500 years later. Disciplines as we know them today formed many centuries later and as such ancient scientists were usually philosophers, mathematicians, and physicists in one. Similar developments were noticeable in other societies as well. In China for instance thinkers like Confucius, Laozi, or Sun Tzu were concerned with question similar to those raised in ancient Greece.

During the following centuries, religion played a major role in the development of knowledge creation. Beliefs about certain essential questions such as how was 
the earth created? where do diseases come from? or what happens after death? impeded scientific advances in many fields and as such slowed down overall knowledge creation. Not very surprisingly, the middle Ages are often considered to be a dark age, in which rational thinking was prohibited. With the invention of the printing press and the beginning of the Renaissance in the 17th century, research slowly emancipated itself from religion. Slowly meaning that it took the church until 1992 to rehabilitate Galileo for his outrageous claim that the sun might be the center of our universe.

During the Renaissance, considerable amounts of knowledge were created by a few polymaths - more or less a small group of outstanding thinkers involved in all kinds of questions ranging from biology, to art, to engineering-hence the label 'Renaissance man'. Da Vinci, for instance, developed machines related to today's helicopters, analyzed water, clouds, and rain, painted some of the most important paintings of mankind, and did considerable research on anatomy. Goethe wrote, did research in botany, and was in dispute with Newton over questions concerning optics and color.

What we consider modern science came to life in the 17th century when knowledge creation was both, professionalized and institutionalized. The number of scientists started to skyrocket-from a few polymath during the renaissance to over a million scientists in 1850. This growth did not slow down over the following 150 years and today we can globally count roughly 100 million people involved in science. More and more disciplines formed and scientists became professional specialists in tiny fields rather than experts in general knowledge.

\section{Professionalization of Knowledge Creation: The First Scientific Revolution}

The professionalization of knowledge creation is often called the first scientific revolution. Indeed it is this revolution that laid the groundwork for many principles that guide scientific work today. Academic disciplines as we today know them formed during the first scientific revolution, as did our publishing system. The professionalization of knowledge creation required means of assessing the value of a contribution, so that incentives for successful research could be provided. Lacking a sufficient system for these incentives, 17th century researchers were secretive in their discoveries. Without a scientific publication system they claimed inventorship by sending out anagrams to fellow researchers that did not make sense without knowledge of the discovery. ${ }^{1}$ This method prevented other scientists from claiming inventorship, and was still a form of publishing. When the knowledge in question began to spread and the anagrams could be made sense of, future research funding was hopefully already secured. Today, this sounds

1 http://www.mathpages.com/home/kmath151/kmath151.htm 
downright preposterous, as we all agree upon the notion that research is always based upon other research and as such that research results should be available to those interested in them.

It was the development of a journal publication system that drastically changed publishing in research and gave appropriate credits to researchers. The first journal purely dedicated to science was Philosophical Transactions which has been published ever since [e.g. one of the first scientific articles (Hook 1665)]. Publishing scientific journal articles became a pivotal building block of modern science. Researchers developed a common understanding that it is the common interest for research results to be openly available to all other researchers (David 2004). This understanding of the necessity for openness in science ... led to the modern journal system, a system that is perhaps the most open system for the transmission of knowledge that could be built with seventeenth-century media (Nielsen 2011). Based on this core concept of publishing, myriads of partially institutionalized, partially commercialized structures grew. These structures developed constitute the cultural, political, and fundamental background in which academic knowledge creation works till today. The entire system is inherently based upon journals printed on paper. Almost every scientific publication we see today is created as if it is meant to be printed. Articles come in very predefined forms and are usually downloaded as printout-like PDFs. There is no fundamental reason to stick to this principle-other than our scientific heritage.

Currently, we can see a transition in knowledge dissemination set off by the Internet that enables scientists to publish in forms unimaginable only a few years ago. In all kinds of disciplines these new methods pop up, be it in the humanities under the term 'digital humanities', from a Web 2.0 angle under the term 'Science 2.0', or from those fighting for free knowledge under the term 'open research' and 'Open Science'. The Internet offers new answers to many challenges which the first scientific revolution overcame hundreds of years ago. And it is the task of today's researchers to assess and evaluate those newly created options, to bridge the legacy gap, and to lay a path towards the second scientific revolution.

\section{Legacy Gap: The Background of the Second Scientific Revolution}

The journal system developed at a time when written or printed letters and a few books were the only means of transferring knowledge. Before printing and disseminating a piece of knowledge, it had to be in a complete and correct form, otherwise it was not worth paying for the costly publication process (Fig. 1). Publishers derived control over scientific content by controlling the printing and dissemination of scientific results. Accordingly, the assessment of scientific impact developed around the journal system.

However, paper is no longer the only media of choice. Publishing costs diminished and from a technical viewpoint preliminary results or idea snippets 


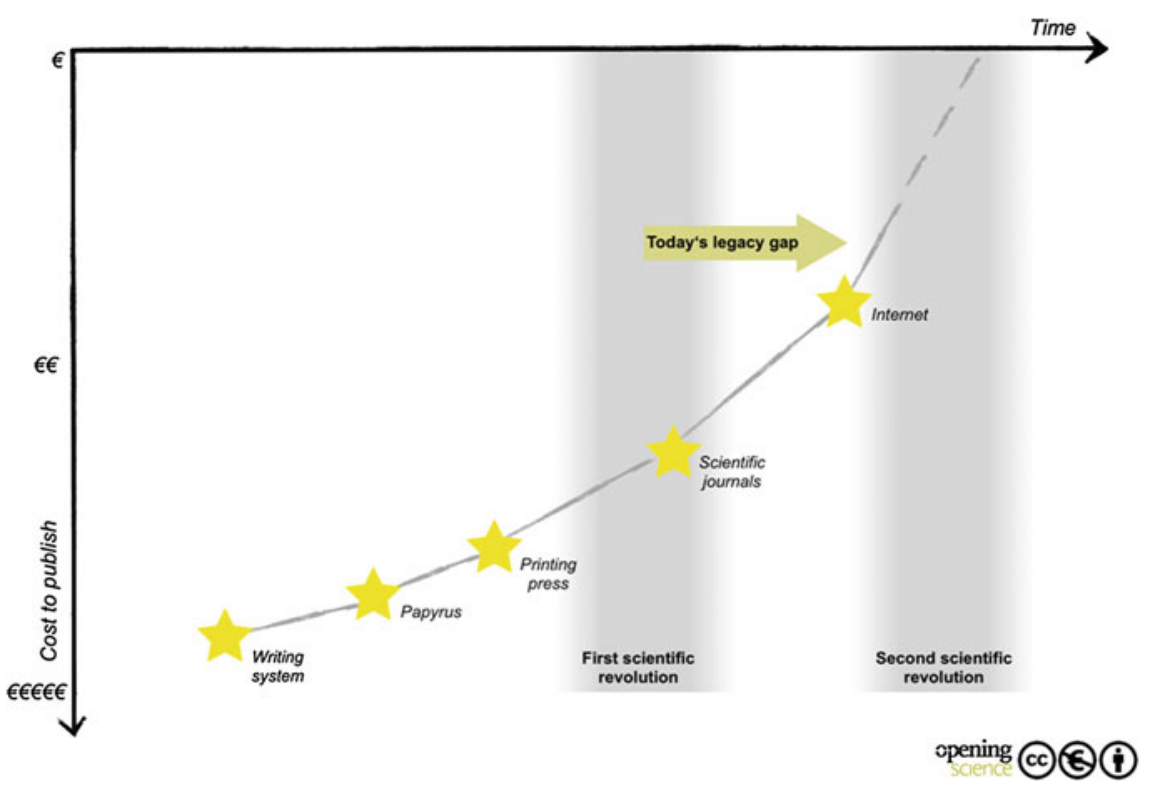

Fig. 1 The first scientific revolution happened when the publishing of scientific papers became the prevailing means of disseminating scientific knowledge. Our scientific culture developed around this. Today the Internet provides novel means of publishing and we are in the 'legacy gap' between the availability of these tools and their profound integration into the scientific culture (second scientific revolution)

could be published, edited, and commented on. Yet, research as a whole is affected by the culture it has developed; it is affected by a the journal system created when results simply had to be printed on paper. We are currently in a "legacy gap" (Fig. 1) and everything points to the fact that we are on the brink of a new scientific revolution. Yet, how this revolution actually will be played out remains one of the most interesting questions in modern science.

\section{The Second Scientific Revolution}

Picture a situation in which scientists would be able to publish all their thoughts, results, conclusions, data, and such as they occur, openly and widely available to everybody. The Internet already provides tools that could make this possible (microblogs, blogs, wikis, etc.). Moreover, picture a scientific culture in which researchers could be in the situation of doing so with the assurance that they will be credited appropriately. Imagine the potential for interactions between researchers. Knowledge could flow quickly, regardless of institutions and personal networks. Research results could be published as they occur. There would be no need to wait until results are complete enough to support a full paper. Similarly, if 

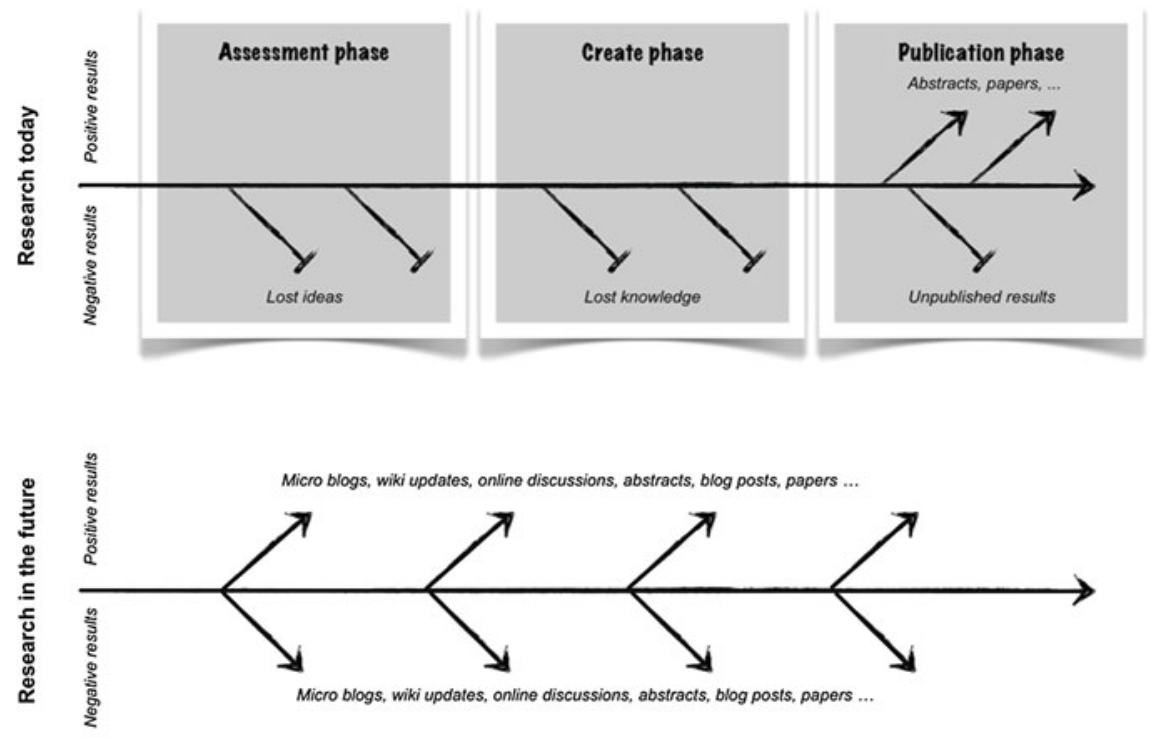

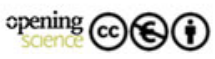

Fig. 2 Today, research projects are conducted until results justify a full-blown paper. In the future, scientists might openly share ideas, preliminary results, and negative results at much earlier stages of their research using the novel publication methods that became available with the Internet

projects were to be stopped, negative or small findings could be published in blog posts or other low threshold publications. These findings could therefore still contribute to the scientific knowledge process. Today, negative results are often dismissed and thus the entire knowledge created in such a research project is not available to others. Someone else might start a similar project running into the same problem that stopped the first project simply because the first project never published an explanation of its failure (Fig. 2).

The advantages of such a scientific culture are multifaceted. We would see faster knowledge exchange, prevention of unnecessarily repeated experiments, and a more vivid discussion (Fig. 3). However, in order to use these novel publication formats, they must be appropriately credited by other scientists and-maybe more importantly_by granting authorities, which is not yet the case.

\section{Naming the New: Science 2.0, Open Science, eScience, Mode2, Open Research}

Terms like Science 2.0, Open Science, Digital Humanities, eScience, Mode2, or Open Research are all umbrella terms that formed over the past few years and that emphasize various aspects of the second scientific revolution. 

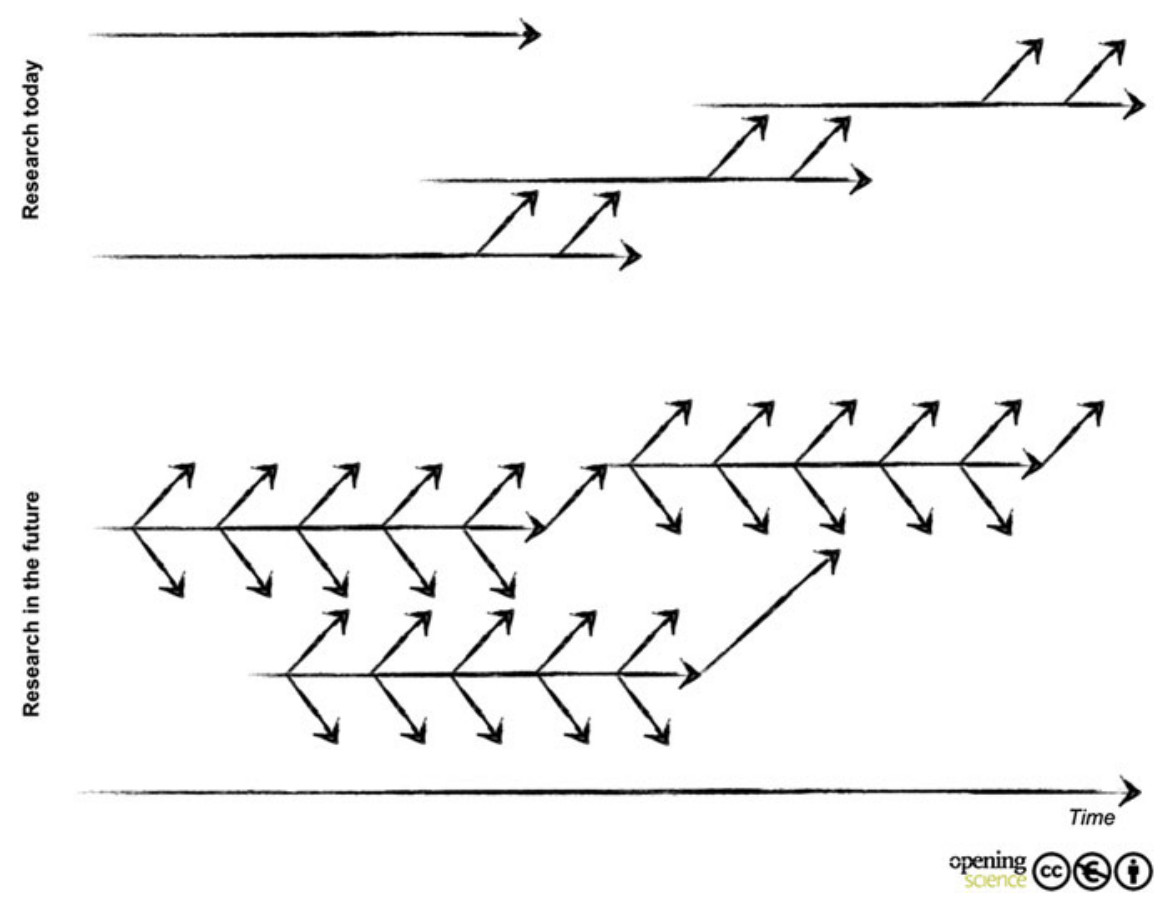

Fig. 3 The research culture of the future possibly supports an open and wide communication beyond institutes and personal networks by providing novel, credited means of disseminating knowledge between researchers. Negative as well as positive findings will contribute to other research projects much sooner after the findings occur

All of these umbrella terms struggle to find a clear definition and people often use them interchangeably when talking about current changes in scientific pursuits. We sought after defining each and every one of these terms in order to establish a coherent picture of how the change in knowledge creation is seen from different angles. Yet, what each of the terms means and how exactly it differs from the others is often unclear. If you ask five people how Mode 2 and Science 2.0 are associated you can be certain to get five different and possibly contradictory answers. All terms are somewhat born of the necessity that a term for the present changes was needed. Knowledge creation is a wide field and thus several terms emerged, whereof we would like to define only two-mainly in order to use them in the discussions contained within this book.

- Science 2.0 refers to all scientific culture, incl. scientific communication, which employs features enabled by Web 2.0 and the Internet (in contrast to Science 1.0 which represents a scientific culture that does not take advantage of the Internet).

- Open Science refers to a scientific culture that is characterized by its openness. Scientists share results almost immediately and with a very wide audience. 


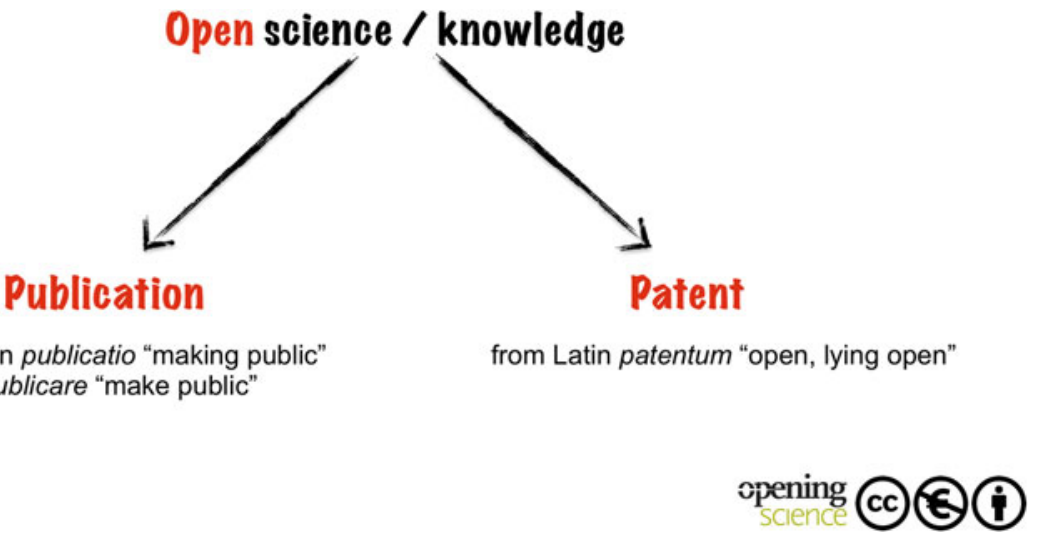

Fig. 4 Since the first scientific revolution, science and knowledge creation was open - as open as the methods of the seventeenth century allowed it to be. The Internet has brought about novel methods, thus allowing science to be more open

Strictly speaking, since the first scientific revolution, science has been open (Fig. 4). Through the Internet and Web 2.0 science can become 'more Open Science', meaning that researchers share results, ideas, and data much earlier and much more extensively to the public than they do at the moment.

Science 2.0 enables Open Science, but Science 2.0 does not necessarily have to happen in an Open Science fashion, since scientists can still employ features of the Internet, but stay very much put in terms of publishing their results. This might be due to cultural and legal restrictions.

\section{The Second Scientific Revolution: Road to a Great New Future?}

Many stakeholders serve the current scientific culture. They brought research, and with it society, quite far. Yet now, we have to face the challenges that come with all the novel developments and with the second scientific revolution. History shows that knowledge creation has always adopted new opportunities. In turn, it certainly will do so this time, too. Yet the question remains as to who will be the drivers and the stakeholders of tomorrow. In the best case, the biggest benefactor will be the scientific knowledge generating process—and with it research itself.

Many researchers show considerable concern in respect to the novel concepts of the second scientific revolution. From these concerns vivid discussions should arise and useful conclusions should be found that steer the second scientific revolution in the right direction. This is especially true since significant input should come from within the active research community itself. 
Another question is whether future openness and onlineness will set optimal incentives for the creation of knowledge. Many wrong paths could be picked and may result in dead-ends. It is important that stakeholders are flexible and honest enough to be able to leave dead-end streets.

Some voices discuss the current transition of research as a revolutionizing process that might overcome current shortcomings in scientific conduct. Shortcomings are among many others: questionable proof generating means (such as wrongly applied statistics (Ioannidis 2005; Sterne 2001), intolerance against uncommon theses and approaches, citation-based 'truth generation', and inflexible cultures of scientific approaches within disciplines. Furthermore, publication-bias through rejection of negative results or rejection of non-confirming studies (Turner et al. 2008; Begley \& Ellis 2012) and questionable incentives that are set by the current methods to assess scientific quality (see chapter Excellence by Nonsense: The Competition for Publications in Modern Science) are also factors. The transition towards the second scientific revolution can help to solve these problems, but it does not necessarily have to. It can be a way to make science more open, liberal, and fair, but it can also result in the opposite.

To conclude, much will depend upon whether researchers become the leading force within this transition, or whether they play a passive role driven by other stakeholders of the research process. In order to prevent the latter, researchers should be deeply involved in this process and they should be aware of the potential consequences. This book is meant to support scientists in becoming a constructing factor in the designing process of the second scientific revolution.

\section{The Second Scientific Revolution is Based on Many Novel Aspects and Tools}

Despite their separation, the key aspects of the second scientific revolution are interconnected (Fig. 5). Open Access (see chapter Open Access: A State of the Art), for instance, needs new forms of copyright concepts (see Creative Commons Licences). Reference managers (see Reference Management) are a great addition to social networks for scientists (see chapter Academia Goes Facebook? The Potential of Social Network Sites in the Scholarly Realm). Assessing the scientific impact of novel publications such as blog posts (see (Micro)Blogging Science? Notes on Potentials and Constraints of New Forms of Scholarly Communication) needs novel impact measurement factors-altmetrics (see chapter Altmetrics and Other Novel Measures for Scientific Impact), which might be based on unambiguous researcher IDs (see chapter Unique Identifiers for Researchers). Altmetrics, at the same time, can be integrated into social networks. There is no single most important factor: it is more a multitude of facets that jointly change how research works. 


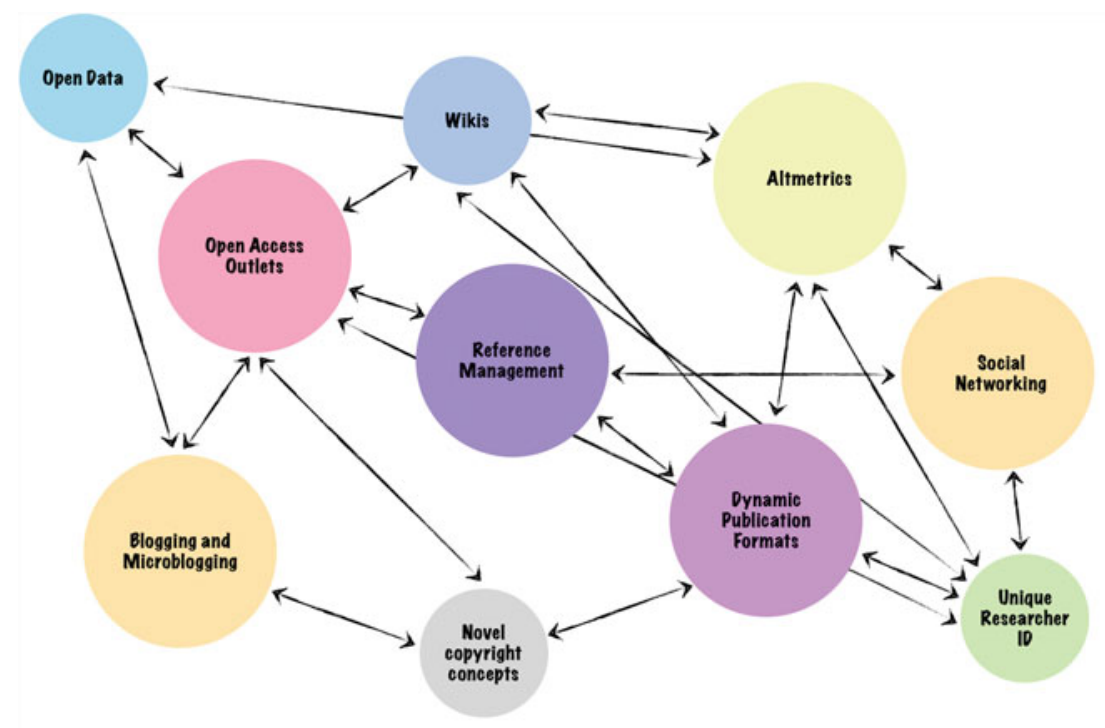

opening @(ㄷ) ()

Fig. 5 It is important to understand that many tools of the second scientific revolution will only make sense if others are also implemented. For example, alternative impact measurement systems such as altmetrics only make sense if researchers can be uniquely identified - either with a Unique Researcher ID or within a social network

\section{How This Book Works: Artificially Dissecting the Second Scientific Revolution}

This book brings together the enabling concepts that shape the current discussion on our changing research environment. We divided the book into three parts in order to make its content easily accessible.

- The first part of the book is called Basics; here we cover topics that highlight the overall shift in scientific thinking. It begins with the chapter "Open Science: One Term, Five Schools of Thought" in which Benedikt Fecher and editor Sascha Friesike explain the many meanings which have been given to the term Open Science. This is followed by Mathias Binswanger's "Excellence by Nonsense: The Competition for Publications in Modern Science" in which he highlights some of the downsides in today publication driven scientific environments. Alexander Gerber's article titled "Science Caught Flat-footed: How Academia Struggles with Open Science Communication" follows; here the author explains why social media are adopted quite slowly by the research community, especially in Europe. The last article in the section was written by Michelle Sidler and is entitled "Open Science and the Three Cultures: 
Expanding Open Science to All Domains of Knowledge Creation"; in it the author highlights a core weakness that the terms Open Science and Science 2.0 share: the fact that all of the implied concepts are valid for researchers outside the sciences as well, yet the name might scare them away.

- The second part of the book is called Tools and deals with implementations that already work today. Cornelius Puschmann starts the section with his piece on blogging and microblogging among researches called "(Micro) blogging Science? Notes on Potentials and Constraints of New Forms of Scholarly Communication". He is followed by Michael Nentwich and René König's article "Academia Goes Facebook? The Potential of Social Network Sites in the Scholarly Realm". "Reference Management" by Martin Fenner, Kaja Scheliga, and editor Sönke Bartling is the next chapter. It is succeeded by "Open Access: A State of the Art" by Dagmar Sitek and Roland Bertelmann, and James MacGregor, Kevin Stranack, and John Willinsky's "The Public Knowledge Project: Open Source Tools for Open Access to Scholarly Communication”.

- The third part named Vision takes a more long term view on the issue and thus explains how single aspects of research might develop over the next decade or two. The section begins with an article by Martin Fenner named "Altmetrics and Other Novel Measures for Scientific Impact" and an article by Lambert Heller, Ronald The, and Sönke Bartling called "Dynamic Publication Formats and Collaborative Authoring". It follows "Open Research Data: From Vision to Practice" by Heinz Pampel and Sünje Dallmeier-Tiessen, and "Intellectual Property and Computational Science" by Victoria Stodden. The next chapter is called "Research Funding in Science 2.0" and was written by Jörg EisfeldReschke, Ulrich Herb, and Karsten Wenzlaff. The last chapter of the book was written by ThomasSchildhauer and Hilger Voss and is entitled "Open Innovation and Crowdsourcing in the Sciences".

- The book closes with a collection of cases which highlight in a rather brief manner some aspects of the Open Science movement. Here the authors focus on specific aspects and projects, give advice, or present their experience

Open Access This chapter is distributed under the terms of the Creative Commons Attribution Noncommercial License, which permits any noncommercial use, distribution, and reproduction in any medium, provided the original author(s) and source are credited.

\section{References}

Begley, C. G., \& Ellis, L. M. (2012). Drug development: raise standards for preclinical cancer research. Nature, 483(7391), 531-533. doi:10.1038/483531a.

Boyd, D. M., \& Ellison, N. B. (2007). Social network sites: definition, history, and scholarship. J. Comput.-Mediated Commun., 13(1), 210-230. doi:10.1111/j.1083-6101.2007.00393.x.

David, P. A. (2004). Understanding the emergence of "Open Science" institutions: functionalist economics in historical context. Ind. Corporate Change, 13(4), 571-589. doi:10.1093/icc/ dth023. 
Hook, M. (1665). Some observations lately made at London concerning the planet Jupiter. Philosophical transactions of the royal society of London, 1(1-22), pp. 245-247. doi:10.1098/ rstl.1665.0103.

Ioannidis, J.P.A. (2005). Why most published research findings are false. PLoS Medicine, 2(8), p. e124. doi:10.1371/journal.pmed.0020124.

Merton, R.K. (1993). On the shoulders of giants: a Shandean postscript Post-Italianate ed., Chicago: University of Chicago Press.

Nielsen, M. (2011). Reinventing discovery: the new era of networked science. New Jersey: Princeton University Press.

Sterne, J. A. C. (2001). Sifting the evidence-what's wrong with significance tests? Another comment on the role of statistical methods. BMJ, 322(7280), 226-231. doi:10.1136/ bmj.322.7280.226.

Turner, E. H., Matthews, A. M., Linardatos, E., \& Tell, R. A. (2008). Selective publication of antidepressant trials and its influence on apparent efficacy. New England J. Med., 358(3), 252-260. doi:10.1056/NEJMsa065779. 